BULL. AUSTRAL. MATH. SOC.

VOL. $26(1982), 161-180$.

\title{
ON THE STRUCTURE OF A CLASS \\ OF EQUIVARIANT MAPS
}

\author{
M.J. FIELD
}

Let $G$ be a compact Lie group and $M$ be a compact $G$-manifold. We investigate the class of equivariant diffeomorphisms of $M$ covering the identity map on the orbit space $M / G$.

\section{Introduction}

Let $H$ be a closed subgroup of the compact lie group $G$. Denote the normaliser and centraliser of $H$ in $G$ by $N(H)$ and $C(H)$ respectively. If we let $h, n(h)$ and $c(h)$ denote the Lie algebras of $H, N(H)$ and $C(H)$ it is an elementary and well known result that $n(h)=h+c(h)$. An immediate consequence is that every element in the identity component $N(H)^{0}$ of $N(H)$ can be written, not necessarily uniquely, as a product ch, where $c \in C(H)^{0}, h \in H^{0}$. The main aim of Section 1 is to provide an alternative, differential geometric proof, of this result. In section 2 we show how our techniques may be used to give information about G-equivariant diffeomorphisms covering the identity map on the orbit space.

We should point out that the techniques and results used in this paper are elementary. In particular, in Section 2, we make no serious use of homotopy theory and stop short of using any of the deeper results of smooth invariant theory as developed by Schwarz in [9].

\section{Centralisers and normalisers}

Let $G$ be a group. Recall that the semi-direct product structure on Received 16 March 1982. 
$G \times G$ is defined by

$$
(a, b) .(c, d)=\left(a c, b a d a^{-1}\right),(a, b),(c, d) \epsilon G \times G .
$$

We have an action of $G \times G$ on $G$ defined by

$$
(a, b) g=b a g a^{-1},(a, b) \in G \times G, g \in G .
$$

From now on we suppose $G$ is a compact Lie group. Using Hasr measure on $G \times G$, we may average any riemannian metric for $G$ over $G \times G$ to obtain a $G \times G$-equivariant riemannian metric on $G$. Let $d($,$) denote$ the corresponding distance function on $G$. Note that if $x, y \in G$ we have

$$
\begin{array}{r}
d(g x, g y)=d(x, y), g \in G, \\
d\left(g x g^{-1}, g y g^{-1}\right)=d(x, y), g \in G .
\end{array}
$$

Indeed, these relations follow immediately from the $G \times G$-invariance of the metric $d$.

LEMMA 1. If $U$ is an open d-disc, centre $e$, in $G$, then

$$
g U g^{-1}=U, g \in G \text {. }
$$

Proof. Immediate from Property (B) of $d$. Compare Bredon [2, Chapter 0, Proposition 1-10].

LEMMA 2. Let $H$ be a closed subgroup of $G$. Then there exists an open d-disc $U$, centre $e$, in $G$ and smooth map $X: U \rightarrow U$ such that:

(1) $x(g) \in g H, g \in U$;

(2) $d(e, g H)=d(e, x(g))$ and $x(g)$ is the unique point in $g H$ satisfying this relation;

(3) $\times\left(h g h^{-1}\right)=h \times(g) h^{-1}, h \in H, g \in U$;

(4) $x(g) \in C(H)$, if $g \in N(H) \cap U$.

Proof. For all $g \in G, g H$ is a submanifold of $G$. Choose a sufficiently small open $d$-disc neighbourhood $U$ of the identity in $G$ such that for every $g \in U$ there.exists a unique minimising geodesic between $e$ and $g H$ (see Bishop and Crittenden [1] and note that our statement amounts to choosing a tubular neighbourhood of $H$ in $G$, relative to the metric $d$ ). Property (A) of $d$ implies that 
$d(e, g H)=d\left(H, g^{H}\right), g \in U$. Define $\chi(g)$ to be the unique point in $g H$ minimising distance between $e$ and $g H$. Note that $\chi(g) \in U$ and that $X(g)$ depends smoothly on $g, g \in U$. By Property (B) of $d$ we have

$$
d(e, g H)=d\left(e, h g H h^{-1}\right)=d\left(e, h \times(g) h^{-1}\right), h \in G \text {. }
$$

But $d\left(e, h g H h^{-1}\right)=d\left(e,\left(h g h^{-1}\right)\left(h H h^{-1}\right)\right)$ and so if $h \in H$ we see that $d\left(e, h g H h^{-1}\right)=d\left(e, h g h^{-1} H\right)$ and so $d\left(e, h \times(g) h^{-1}\right)=d\left(e, x\left(h g h^{-1}\right)\right)$. By uniqueness of $X$, it follows that

$$
\chi\left(h g h^{-1}\right)=h \times(g) h^{-1}, h \in H, g \in U .
$$

Now if $g \in N(H) \cap U, h g h^{-1} H=g H$ and so

$$
h \times(g) h^{-1}=\chi(g), h \in H, g \in N(H) \cap U,
$$

proving (4).

COROLLARY TO LEMMA 1. The identity component of $N(H)$ is generated by the identity components of $H$ and $C(H)$. Moreover, $C(H)$ meets $H$ transversally at $e$, in $N(H)$.

Proof. Immediate from (4) of Lemma 2.

LEMMA 3. If $H_{1}, \ldots, H_{p}$ are closed subgroups of $G$ then there exists on open $d$-disc $U$, centre $e$, in $G$ such that for all $g \in U \cap N\left(H_{j}\right), j=1, \ldots, p$, there exists a unique $x(g) \in g H_{j} \cap c\left(H_{j}\right)$ minimising distance between $e$ and $g_{j}{ }_{j}$. Moreover, if $k \in G$, $g \in U \cap N\left(H_{j}\right), \quad k \times(g) k^{-1} \in\left(k g k^{-1}\right)\left(k H_{j} k^{-1}\right) \cap C\left(k H_{j} k^{-1}\right) \cap U$ and is the wique point minimising distance between $e$ and $\mathrm{kgH}_{j} \mathrm{k}^{-1}$.

Proof. The first part follows by choosing a sufficiently small disc at $e$ which works for $H_{1}, \ldots, H_{p}$. The second part is immediate from Property (B) of the metric together with the invariance of $U$ under the adjoint action (Lemma 1).

REMARK. Let $\Sigma$ denote the cut locus of the exponential map of $h^{\perp} \subset n(h)$ in $G$ (see Bishop and Crittenden [1, pp. 237-241]). It is well known that $\Sigma$ is always a sphere (topologically) though $\Sigma$ may have 
singularities. Two questions naturally arise. Are the singularities of $\Sigma$ always rational? To what extent can we regard the singularities of $\Sigma$ as obstructions to expressing $N(H)^{0}$ as a product of $H^{0}$ and $C(H)^{0}$ and how might these singularities relate to cohomological invariants of this extension problem?

\section{Structure of equivariant diffeomorphisms which are trivial mod $G$}

Let $M$ be a compact $G$-manifold. Throughout this section we assume $M$ is connected and $G$ acts smoothly on $M$ ("smooth" will always mean $C^{\infty}$ ). We follow the notation of Bredon [2] (see also Field [3, section 1]). Thus if $x \in M, G(x)$ will denote the G-orbit through $x$ and $G_{x}$ the isotropy subgroup of $G$ at $x$. We partition $M$ into points of the same $G$-orbit type and write

$$
M=\bigcup_{i=1}^{N} M_{i},
$$

where all points in $M_{i}$ have the same $G$-orbit type and if there exists $x \in M_{i}, y \in M_{j}$ such that $G_{x} \supsetneqq G_{y}$ then $i<j$. We refer to $M_{N}$ as the principal G-orbit type and remark that $M_{N}$ is open and dense in $M$ and $M_{N} / G$ is connected.

Let $F^{r}(M)$ denote the group of $c^{r}$ equivariant diffeomorphisms of $M$ satisfying $f(x) \in G(x)$ for all $x \in M$. Give $F^{r}(M)$ the $C^{r}$ topology. We say that a $C^{r}$ map $x: M \rightarrow G$ is $c^{r}$ skew G-equivariant if

$$
\chi(g x)=g X(x) g^{-1}, g \in G, x \in M .
$$

Denote the set of $C^{r}$ skew equivariant maps of $M$ into $G$ by $S^{r}(M)$. Observe that $S^{r}(M)$ has the structure of a group with composition defined by

$$
\left(X_{1} \cdot X_{2}\right)(x)=X_{1}(x) X_{2}(x),
$$

$\chi_{1}, \chi_{2} \in S^{r}(M), x \in M$. We have a natural group homomorphism 
$\gamma: S^{r}(M) \rightarrow F^{r}(M)$ defined by

$$
Y(X)(x)=X(x) x, \quad x \in M .
$$

In future we write $\gamma(\chi)=\hat{\chi}, \quad x \in S^{r}(M)$ and set $\Gamma^{r}(M)=\gamma\left(S^{r}(M)\right)$.

LEMMA 1. $\Gamma^{r}(M)$ is a normal subgroup of $F^{r}(M)$.

Proof. Let $x \in S^{r}(M), f \in F^{r}(M)$. Then

$$
\left(f^{-1} \hat{\chi} f\right)(x)=\chi(f(x)) x, \quad x \in M
$$

Defining $B \in S^{r}(M)$ by $\beta(x)=\chi(f(x))$, we see that

$$
f^{-l} \hat{\chi} f=\hat{\beta} \in \Gamma^{r}(M)
$$

and so $\Gamma^{r}(M)$ is normal in $\frac{r}{F^{r}}(M)$.

We let $F_{0}^{r}(M)$ denote the subgroup of $F^{r}(M)$ consisting of diffeomorphisms which are $c^{r}$ equivariantly isotopic to the identity (through elements of $F^{r}(M)$ ). Set $\Gamma_{0}^{r}(M)=F_{0}^{r}(M) \cap \Gamma^{r}(M)$.

The main aim of this section is to investigate the group $F^{r}(M) / \Gamma^{r}(M)$ and, in particular, find conditions which allow us to assert that an element of $F^{r}(M)$ actually lies in $\Gamma^{r}(M)$. First, however, we shall prove a useful technical lemma and then give some examples.

LEMMA 2. Let $G$ be a finite group and $f$ be a homeomorphism of the compact G-manifold $M$ (we do not assume $f$ is equivariant). Suppose $f(x) \in G(x)$ for all $x \in M$. Fix $z \in M_{N}$ and suppose $f(z)=g z$, some $g \in G$. Then $f(x)=g x$ for all $x \in M$.

Proof. Let $X=\{x \in M: f(x)=g x\}$. Obviously $X$ is a closed, non-empty, subset of $M$. Since $G$ is finite and $f$ is continuous $f(x)=g x$ for all $x$ in some open neighbourhood of $z$ contained in $M_{N}$. Therefore $X \cap M_{N}$ is open and closed in $M_{N}$ and so $X \cap M_{N}$ is a union of connected components of $M_{N}$. If $M_{N}$ is connected it follows that $X \supset M_{N}$ and so, since $M_{N}$ is dense in $M, X=M$. Suppose $M_{N}$ is not connected 
and denote a connected component of $M_{N}$ contained in $X$ by $M_{N}^{\alpha}$. Set $M_{N}^{b}=f\left(M_{N}^{a}\right)$. By considering $f^{-1}$ we see that $M_{N}^{b}$ is also a connected component of $M_{N}$. Since $M_{N}$ is not connected there exist orbit types $M_{P}$ of codimension one in $M$. Denote the union of the codimension 1 orbit types by $P$. Let $y \in P \cap \vec{M}_{N}^{a}$ and denote the other connected component of $M_{N}$ whose closure contains $y$ by $M_{N}$. Locally, at $y, G_{y}$ acts as reflections in $P$. Let $r \in G_{y}$ denote such an element which acts as a local reflection in $P$. Choose a slice $U$ for the G-action at $y$. Since $y \in X$ and $f$ is continuous we may find $x \in M_{N}^{f} \cap U$ such that $f(x) \in g(U)$. Either $f(x)=g x$ or $f(x)=g r x$. But if $f(x)=g r x$ we must have $f(x) \in M_{N}^{b}$, contrary to the bijectivity of $f$. Hence $f(x)=g x$ and so $x \in X$. Therefore $M_{N}^{\mathcal{C}} \subset X$. It follows that $X \supset M_{N}$ and so $X=M$.

EXAMPLE 1. Let $G$ be a finite group acting smoothly on the compact manifold $M$. Denote the principal orbit type of $M$ by $M_{N}$. Since $G$ is finite $G_{x}$ is independent of $x \in M_{N}$ and we set $H=G_{x}, x \in M_{N}$. Note that $H$ is a normal subgroup of $G$. For $g \in G$ we let $[G, g]$ denote the set $\left\{h^{-1} g^{-1} h g: h \in G\right\}$. Define $\tilde{P}=\{g \in G:[G, g] \subset H\}$. It is easily verified that $\tilde{P}$ is a subgroup of $G$ containing $H$ as a normal subgroup. Set $P=\tilde{P} / H$. We claim that $F^{r}(M) \approx P, 0 \leq r \leq \infty$, and that $F^{r}(M)=\Gamma^{r}(M), 0 \leq r \leq \infty$. Suppose $\alpha \in \tilde{P}$. Define $f_{\alpha} \in F^{\infty}(M)$ by $f_{\alpha}(x)=\alpha x, x \in M$. Since $\alpha \in \tilde{P}$, it is clear that $f_{\alpha}$ is equivariant. Moreover, since $H \subset G_{x}$ for all $x \in M, f_{\alpha}$ depends only on the class of $\alpha$ in $P$. Hence we have defined a map of $P$ into $E^{\infty}(M)$ which is clearly a group homomorphism. Next suppose $f \in \Gamma^{0}(M)$. Fix $z \in M_{N}$. Then $f(z)=g z$ for some $g \in G$. Since $G$ is finite and $f$ is continuous, Lemma 2 implies that $f(x)=g x$ for all $x \in M$. Since $f$ is equivariant it follows easily that $g \in \tilde{P}$ and so we have constructed a map 
of $\Gamma^{0}(M)$ into $P$. This map is obviously the inverse of the homomorphism constructed above and so $F^{\infty}(M)=\Gamma^{0}(M) \approx P$, proving our assertions.

EXAMPLE 2. Let $S^{\mathcal{I}}=[0,2 \pi] / 0=2 \pi$ act on $\mathbb{C}$ in the standard way as multiplication by $e^{i \theta}$. Suppose $f \in F^{r}(\mathbf{C})$. In case $r=\infty$, Schwartz' theorem on smooth invariants [8] allows us to write $f(z)=g\left(|z|^{2}\right) z$ for some smooth map $g: \mathbf{R} \rightarrow \mathbb{C}$. Since $\left|g\left(|z|^{2}\right)\right|=1$ for all $z \in \mathbb{C}$, we may define $\chi \in S^{\infty}(\mathbb{C})$ by $\chi(z)=g\left(|z|^{2}\right)$ and then $f=\hat{x}$. Thus we have proved that $F^{r}(\mathbf{C})=\Gamma^{r}(\mathbb{C})$ in case $r=\infty$. This result is definitely false if $r<\infty$. For example, if we define

$$
\begin{aligned}
f(z) & =\exp \left(i /|z|^{2}\right) z, \quad z \neq 0, \\
& =0, \quad z=0,
\end{aligned}
$$

we see that $f \in F^{0}(\mathbb{C})$ but that $f$ is not $C^{\perp}$. Clearly $f \notin \Gamma^{0}(\mathbb{C})$. Similar examples show that $\vec{F}^{r}(\mathbb{C}) \neq \Gamma^{2}(\mathbb{C}), 0 \leq r<\infty$.

EXAMPLE 3. Parametrize the torus $T^{2}=S^{l} \times S^{l}$ by $(\theta, \psi) \in[0,2 \pi] \times[0,2 \pi]$ and take the $S^{1}$-action on $T^{2}$ defined by $e^{i \sigma}(\theta, \psi)=(\theta, \psi+2 \sigma), \bmod 2 \pi$.

Define $f: T^{2} \rightarrow T^{2}$ by

$$
f(\theta, \psi)=(\theta, \psi+\theta), \bmod 2 \pi
$$

Clearly $f \in F^{\infty}\left(T^{2}\right)$ but $f \neq F_{0}^{\infty}\left(T^{2}\right)$. Notice that we cannot write $f=\hat{\chi}$ for some $x \in S^{\infty}\left(T^{2}\right)$ since $f(\theta, \psi)=e^{i \theta / 2}(\theta, \psi)$. In fact it is not hard to verify that $F^{\infty}\left(T^{2}\right) / \Gamma^{\infty}\left(T^{2}\right) \cong \mathbb{Z}_{2}$ (the isotropy group of the $S^{1}$-action) and $\Gamma^{\infty}\left(T^{2}\right) / \Gamma_{0}^{\infty}\left(T^{2}\right) \cong \mathbb{Z}$ (the first cohomology group of the orbit space).

EXAMPLE 4. Regard $S U(2)$ as the group of complex matrices

$$
\left(\begin{array}{cc}
a & b \\
-\bar{b} & \bar{a}
\end{array}\right), a, b \in \mathbb{C},
$$


subject to $|a|^{2}+|b|^{2}=1$, and take the corresponding matrix representation of $S U(2)$ on $\mathbf{c}^{2}$. Let $A \in$ End $\left(\mathbb{C}^{2}\right)$ be scalar multiplication by $e^{i \theta}, \theta \in(0,2 \pi)$. Certainly $A$ is an $S U(2)-$ equivariant map; indeed $A \in F^{\infty}\left(\mathbb{C}^{2}\right)$. Let us try to find a skew $S U(2)-$ equivariant map $\chi: \mathbb{C}^{2} \rightarrow S U(2)$ such that $A=\hat{\chi}$. If we fix $z=\left(z_{1}, z_{2}\right) \neq 0$ and solve

$$
A(z)=\left(\begin{array}{cc}
a & b \\
-\bar{b} & \bar{a}
\end{array}\right)(z)
$$

for $a$ and $b$ we find

$$
\begin{aligned}
& a(z)=\left(e^{i \theta}\left|z_{1}\right|^{2}+e^{-i \theta}\left|z_{2}\right|^{2}\right) /\|z\|^{2}, \\
& b(z)=\left(z_{1} \bar{z}_{2}\left(e^{i \theta}-e^{-i \theta}\right)\right) /\|z\|^{2},
\end{aligned}
$$

where $\|z\|^{2}=\left|z_{1}\right|^{2}+\left|z_{2}\right|^{2}$. Suppose $\theta \neq \pi$. If we define

$$
x(z)=\left(\begin{array}{cc}
a(z) & b(z) \\
-\overline{b(z)} & \overline{a(z)}
\end{array}\right), \quad z \neq 0,
$$

we see that the resulting uniquely determined skew $S U(2)$-equivariant map $x$ does not extend continuously to $\mathbb{C}^{2}$. Consequently $F^{\infty}\left(\mathbb{C}^{2}\right) / \Gamma^{\infty}\left(\mathbb{C}^{2}\right)$ is infinite. Let us examine what happens if we use the polar blowing-up construction described in Field [4]. Recall that the polar blowing-up of $\mathbb{C}^{2}$ at 0 is the principal $S U(2)$-manifold $S^{3} \times \mathbf{R}$ together with the projection $\pi: S^{3} \times \mathrm{R} \rightarrow \mathrm{C}^{2}$ defined by $\pi(u, t)=t u$ (we regard $s^{3} \subset c^{2}$. Note that here we take $\pi(u, t)=t u$ rather than $t^{2} u$ as was done in Field [4]). The map $A$ lifts to $\tilde{A}: S^{3} \times \mathbb{R} \rightarrow S^{3} \times \mathbb{R}$ where $\tilde{A}(u, t)=\left(e^{i \theta} u, t\right)$. Therefore if $u=\left(z_{1}, z_{2}\right) \in S^{3}$ we see that

$$
A(u, t)=\left(\left(\begin{array}{cc}
a(u) & b(u) \\
-\overline{b(u)} & \overline{a(u)}
\end{array}\right)(u), t\right)
$$

where $a(u), b(u)$ are as constructed explicitly above. But $a, b$ 
restrict to smooth maps on $S^{3}$ and so we see that $\tilde{A} \in \Gamma^{\infty}\left(s^{3} \times \mathbb{R}\right)$. If we write $\tilde{A}=\hat{\mu}, \mu \in S^{\infty}\left(S^{3} \times \mathbb{R}\right)$, then we may define a skew $S U(2)$ equivariant map $x: c^{2} \rightarrow S U(2)$ by

$$
x(z)=\mu(y),
$$

where $y$ is any point in $\pi^{-1}(y)$. Although the map $\chi$ is not continuous (at 0 ) nevertheless we do have $A=\hat{x}$.

THEOREM 1. Let $G$ be a compact Lie group acting smoothly on the compact differential manifold $M$. Suppose that all G-orbits are of the same dimension. Then

$$
\begin{aligned}
& \text { (1) } \Gamma_{0}^{r}(M)=F_{0}^{r}(M), 0 \leq r \leq \infty, \\
& \text { (2) } F^{r}(M) / \Gamma^{r}(M) \text { qs independent of } r, 0 \leq r \leq \infty, \\
& \text { (3) } F^{r}(M) \cong I S F^{r}(M) \times \Gamma_{0}^{r}(M),
\end{aligned}
$$

where $\operatorname{ISH}^{r}(M)$ denotes the group of isotopy classes of $c^{r}$ equivariant diffeomorphisms in $F^{r}(M)$ (isotopies through elements of ${ }_{F}^{r}(M)$ ).

The proof of this result will be broken into a number of lemmas.

LEMMA A. Given any open neighbourhood $U$ of the identity in $G$ and $0 \leq r \leq \infty$, there exists an open neighbourhood $N$ of the identity in $F^{r}(M)$ such that if $f \in N$ there exists a $c^{r}$ map $\gamma: M \rightarrow G$ such that for all $x \in M$ we have $f(x)=\gamma(x) x$.

Proof. Using slices we may reduce the proof of Lemma $A$ to the case when $M=G \times{ }_{H} D$, where $D$ is a closed disc, centre 0 in an $H-$ representation $V$ and all H-orbits in $V$ have dimension zero. The proof of this special case may be found in Field [6, Lemma B].

REMARK. If the dimension of G-orbits varies, Lemma A will only be valid for $r \geq r_{0}$, where $r_{0}$ is some positive integer less than the number of distinct G-orbit types of $M$. This may easily be seen using the blowing-up techniques of Field [4] together with the method of proof of Lemma $A$. In general we cannot take $r_{0}=0$ - the $S^{1}$-action of Example 3 
provides a suitable counterexample.

LEMMA B. There exists a neighbourhood $N$ of the identity in $F^{r}(M), 0 \leq r \leq \infty$, such that if $f \in N$ there exists $\chi \in S^{r}(M)$ such that $f=\hat{x}$.

Proof ( $c f$. Field [6, Lemma c]). As in Section 1 we take the semidirect product structure on $G \times G$ and a $G \times G$-equivariant riemannian metric $d$ on $G$. Fix an equivariant riemannian metric on $M$. Let $x \in M$ and set $G_{x}=H$. Then $G(x)$ has an open tubular neighbourhood $W$ smoothly equivariantly diffeomorphic to $G \times{ }_{H} V$ where $V$ is the orthogonal complement of $T_{x} G(x)$ in $T_{x} M$. In future we regard $W$ as identified with $G \times{ }_{H} V$. Since all G-orbits are assumed to have the same dimension every orbit of the action of $H$ on $V$ is finite. Let $H^{0}$ denote the identity component of $H$. If $y \in V$ then $H^{0} \subset H_{y} \subset H$ and so there exist only finitely many distinct isotropy groups for the action of $H$ on $V$ say $H=K_{1}, \ldots, K_{P}$, where $K_{P}$ denotes the principal isotropy group of the action of $H$ on $V$. As in Section 1 we may find for $I \leq j \leq P$ a closed neighbourhood $U_{j}$ of the identity in $G$ such that for $g \in U_{j}$ there exists a unique minimising geodesic between $e$ and $g K_{j}$. Clearly we may assume $U_{1}=\ldots=U_{p}=U$ and that $U$ is a closed $d$-disc neighbourhood of the identity in $G$. For $g \in U$, $d\left(e, g K_{j}\right)=d\left(e, k_{j}(g)\right)$ for a unique $k_{j}(g) \in g K_{j} \cap U$. Since the groups $K_{j}$ have common identity component $H^{0}$ it is clear that we may require $U$ chosen sufficiently small so that $k_{1}=\ldots=k_{P}=k$, say. The map $k: U \rightarrow U$ is clearly smooth. By Lemma A, there exists a neighbourhood $N_{W}$ of the identity in $F^{r}(M)$ and a $C^{r}$ map $\gamma: M \rightarrow U$ such that $f(z)=\gamma(z) z$ for all $f \in N_{W}, z \in M$. We define $\tilde{X}_{X}: V \rightarrow G$ by $x_{W}(y)=k(\gamma(y)), y \in V$. Certainly $\tilde{x}_{W}$ is $c^{r}$ and, by Section $1, \tilde{x}_{W}$ is skew H-equivariant. Extending. $\tilde{X}_{W}$ equivariantly to $W$ we have shown 
that $f \mid W=\hat{\chi}_{W}$ for some $\chi_{W} \in S^{r}(W)$. Now take a finite cover of $M$ by $G$-tubular neighbourhoods $W_{1}, \ldots, W_{m}$. Set $\chi_{W_{i}}=x_{i}, N_{W_{i}}=N_{i}$, $i=1, \ldots, m$. Let $N=\bigcap_{i=1}^{m} N_{i}$. Given $y \in w_{i} \cap w_{j}, f \in N$, suppose $f(y)=g y$. Since $\chi_{i}(y)$ is characterised as being the unique point in ${ }^{g H}{ }_{y}$ minimising distance between $H_{y}$ and ${ }^{H} y_{y}$ it follows that $x_{i}(y)=x_{j}(y)$. Hence we may define $x \in S^{r}(M)$ by $x \mid w_{i}=x_{i}$, $i=1, \ldots, m$. clearly $f=\hat{x}$.

LEMMA $C$. Let $f \in F_{0}^{r}(M), r \geq 0$. Then there exists $x \in S^{r}(M)$ such that $f=\hat{x}$.

Proof. Follows easily from Lema $B$ and we omit details (see the proof of Lemma $D$ in Field [6]).

Proof of Theorem 1. Suppose $f \in F_{0}^{r}(M)$. Then by Lemma $C$ there exists $x \in S^{r}(M)$ such that $f=\hat{\chi}$. Hence $F_{0}^{r}(M) \subset \Gamma_{0}^{r}(M)$. Since the reverse inclusion is obvious we have proved statement (1) of the theorem. Statement (3) follows by observing that if $f, f^{\prime} \in F^{p}(M)$ determine the same element of $I S F^{r}(M)$ then $f^{\prime} f^{-1} \in \frac{F_{0}^{r}}{0}(M)$. Statement (2) follows from (3) once we have shown that the group $I S F^{r}(M)$ is independent of $r$. For this it is enough to show that every element of $\operatorname{ISF}^{p}(M)$ can be represented by a smooth equivariant diffeomorphism. The proof of this assertion is easily accomplished by localising using an equivariant partition of unity, applying Wassermann's approximation theorem [10] and using the fact that $G$-orbits all have the same dimension. We omit the tedious details.

We now analyse the structure of the groups $F^{r}(M), \Gamma^{r}(M)$ a little more closely. We continue to assume that all Gorbits have the same dimension. 
LEMMA 3. Let $f \in F^{r}(M)$ and suppose that for some $z \in M_{N}$, $f(z) \in N\left(G_{z}\right)^{0}(z)$. Then $f(x) \in N\left(G_{x}\right)^{0}(x)$ for alz $x \in M$.

Proof. Using Lemma 2 it follows easily that $f(x) \in N\left(G_{x}\right)^{0}(x)$ for all $x \in M_{N}$. The result now follows from Lemma $E$ of Field [6].

For $r \geq 0$, set $\hat{F}^{r}(M)=\left\{f \in F^{r}(M): f(x) \in N\left(G_{x}\right)^{0}(x)\right.$, all $\left.x \in M\right\}$ and $\hat{\Gamma}^{r}(M)=\Gamma^{r}(M) \cap \hat{F}^{r}(M)$. It is easily verified that $\hat{F}^{r}(M), \hat{\Gamma}^{r}(M)$ are normal subgroups of $E^{r}(M), \Gamma^{r}(M)$ respectively.

PROPOSITION 1. The group $\vec{F}^{r}(M) / \hat{F}^{r}(M)$ is finite and independent of $r \geq 0$. It has order less than or equal to the order of $N(H) / H \cdot N(H)^{0}$ where $H$ denotes any principal isotropy group of the action of $G$ on $M$. In particular, if $G$ is abelian

$$
F^{r}(M) / \hat{F}^{r}(M) \approx G / K \cdot G^{0}
$$

where $K$ denotes the principal isotropy group of the action of $G$ on $M$.

Proof. First observe that if $z \in M$ then $N\left(G_{z}\right)(z) \cong N\left(G_{z}\right) / G_{z}$. Moreover, since $\left(N\left(G_{z}\right) / G_{z}\right)^{0} \approx N\left(G_{z}\right)^{0} / G_{z} \cap N\left(G_{z}\right)^{0}$, we have $N\left(G_{z}\right)^{0}(z) \cong\left(N\left(G_{z}\right) / G_{z}\right)^{0}$. Suppose $f, f^{\prime} \in F^{r}(M)$. If there exists $z \in M_{N}$ such that $f(z), f^{\prime}(z)$ belong to the same connected component of $N\left(G_{z}\right)(z)$ then $f^{\prime} f^{-1}(z) \in N\left(G_{z}\right)^{0}(z)$ and so, by Lemma 3 , $f^{\prime} f^{-1}(x) \in N\left(G_{x}\right)^{0}(x)$ for all $x \in M$. It follows immediately that $F^{r}(M) / \hat{F}^{r}(M)$ is finite with order bounded by the order of $(N(H) / H) /(N(H) / H)^{0}$, where $H$ denotes any principal isotropy group of the action of $G$ on $M$. But

$$
\begin{aligned}
&(N(H) / H) /(N(H) / H)^{0} \approx(N(H) / H) /\left(N(H)^{0} / H \cap N(H)^{0}\right) \approx(N(H) / H) /\left(H \cdot N(H)^{0} / H\right) \\
& \approx N(H) / H \cdot N(H)^{0} .
\end{aligned}
$$

The assertion about the independence of $r$ of the quotient group follows 
using Wassermann's approximation theorem as in the proof of Theorem 1. We leave the remaining statement to the reader.

Let $H$ denote a principal isotropy subgroup of the action of $G$ on $M$ and $M^{H}$ denote the fixed point set of $H$. Let $M^{\langle H\rangle}$ denote the set of points in $M$ with isotropy group $H$ and $\tilde{M}^{H}$ denote the closure of $M^{\langle H\rangle}$. Observe that $M^{H}, \tilde{M}^{H}$ have the structure of compact $N(H)$-manifolds and that $\tilde{M}^{H}$ is a union of connected components of $M^{H}$ (see Schwartz [9, Theorem 11.6]). We let $S^{r}\left(\tilde{M}^{H}\right)$ denote the group of $C^{r}$ skew $N(H)$ equivariant maps of $\tilde{M}^{H}$ into $N(H) / H$.

PROPOSITION 2. Let $M$ be a compact G-manifold and suppose that all $G$-orbits have the same dimension. Then for $r \geq 0$ we have canonical isomorphisms

$$
F^{r}(M) \approx F^{r}\left(\tilde{M}^{H}\right) \approx S^{r}\left(\tilde{M}^{H}\right),
$$

where $F^{r}\left(\tilde{M}^{H}\right)$ denotes the set of $C^{r} \quad N(H)$-equivariant diffeomorphisms of $M^{H}$ covering the identity map of the orbit space.

Proof. Suppose, $\mu \in S^{r}\left(\tilde{M}^{H}\right)$. Then $\mu(n x)=n \mu(x) n^{-1}$ for all $x \in \tilde{M}^{H}, n \in N(H)$. Define $\tilde{f}_{\mu}: \tilde{M}^{H} \rightarrow \tilde{M}^{H}$ by $\tilde{f}_{\mu}(x)=\mu(x) x$. Certainly $\tilde{f}_{\mu}$ is $c^{r}$. Moreover, $\tilde{f}_{\mu}$ is $N(H)$-equivariant since for $x \in \tilde{M}^{H}$, $n \in N(H)$ we have

$$
\begin{aligned}
\tilde{f}_{\mu}(n x) & =\mu(n x) n x \\
& =n \mu(x) n^{-1} n x=n \mu(x) x \\
& =n \tilde{f}_{\mu}(x) .
\end{aligned}
$$

Suppose $g x=h y, x, y \in \tilde{M}^{H}, g, h \in G$. Then $h^{-1} g \in N(H)$. How $\tilde{g \tilde{f}_{\mu}}(x)=h h^{-1} g \tilde{f}_{\mu}(x)=h \tilde{f}_{\mu}\left(h^{-1} g x\right)=h \tilde{f}_{\mu}(y)$. Hence we may extend $\tilde{f}_{\mu}$ to a $c^{p}$ equivariant diffeomorphism $f_{\mu}$ of $M$ by setting $f_{\mu}(g x)=g \tilde{f}_{\mu}(x)$, $x \in \tilde{H}^{H}, g \in G$. Obviously $f_{\mu} \in F^{r}(M)$ and so we have constructed a 
homomorphism $\mu \mapsto f_{\mu}$ of $S^{r}\left(\tilde{M}^{H}\right)$ into $\vec{F}^{r}(M)$ which factors through $F^{r}\left(\tilde{M}^{H}\right)$. Conversely, suppose $f \in F^{r}(M)$. Denote the restriction of $f$ to $\tilde{M}^{H}$ by $\tilde{f}$. Thus $\tilde{f} \in F^{p}\left(\tilde{M}^{H}\right)$. For $x \in M^{\langle H\rangle}, \tilde{f}$ determines a unique $C^{r}$ skew $N(H)$-equivariant map $\gamma_{f}: M^{\langle H\rangle} \rightarrow N(H) / H$ by $\tilde{f}(x)=\gamma_{f}(x) x$. We claim $\gamma_{f}$ extends uniquely to $\tilde{M}^{H}$ as a $C^{r}$ skew $N(H)$-equivariant map. Fix $x \in \tilde{N}^{H}$ and choose a slice $S$ at $x$. Let $\tilde{f}(x)=g x$, some $g \in N(H)$. Then $g^{-1} \tilde{f}(x)=x$ and $g^{-1} \tilde{f}: \tilde{M}^{H} \rightarrow \tilde{M}^{H}$ covers the identity map on the orbit space $\tilde{M}^{H} / N(H)$. Choose a $C^{\infty}$ local section $\omega$ of $N(H)$ over some open neighbourhood $U$ of the identity coset in $N(H) / N(H)_{x}$. Shrinking $S$ if necessary we may assume that $\omega(U)(S) \supset\left(g^{-1} \tilde{f}\right)(S)$. Fix $x \in M^{\langle H\rangle} \cap S$. Now $g^{-1 \tilde{f}(z) \in \omega(U)(N(H)} x^{(z))}$. If $g^{-1 \tilde{f}(z) \notin \omega(U)(z)}$, choose $k \in N(H) x$ so that $k g^{-1 \tilde{f}(z) \in \omega(U)(z)}$. As in the proof of Lemma 2 it then follows that $\mathrm{kg}^{-1} \tilde{f}(y) \in \omega(U)(y)$ for all $y \in S$. Hence, by the implicit function theorem, there exists a $c^{r}$ map $\pi: S \rightarrow N(H)$ such that $k g^{-1} \tilde{f}(y)=\pi(y) y, y \in S$. Regarding $\pi$ as a map into $N(H) / H$, we see that $\pi=\gamma_{f}$ on $M^{\langle H\rangle} \cap S$. Extending $\pi$ $N(H)$-equivariantly to $N(H)(S)$, we see that $\gamma_{j} \mid M^{\langle H\rangle} \cap N(H) S$ extends to a $C^{r} N(H)$-skew equivariant map from $M(H) S$ to $N(H) / S$. Since $x \in \tilde{M}^{H}$ was chosen arbitrarily we have shown that $\gamma_{f}$ extends to all of $\tilde{M}^{H}$ as a $C^{p}$ skew $N(H)$-equivariant map. Clearly $f \mapsto \gamma_{f}$ is the inverse of the map $\mu \rightarrow f_{\mu}$ constructed above.

Continuing with our assumption that $H$ is a principal isotropy group of the action of $G$ on $M$ we note that, in general, $N(H) \neq C(H)$.H. It is true, however, that $N(H) \supset C(H) . H$ and $N(H)^{0}=C(H)^{0} . H^{0}$, the latter statement following from Section 1 . We let $\alpha: C(H) \rightarrow N(H) / H$, B : $C(H)^{0} \rightarrow N(H)^{0} / H \cap N(H)^{0}$ denote the associated projection maps and 
remark that $\alpha$ is generally not onto whilst $\beta$ is always onto. We have an $N(H)$-action on $C(H)$ ( $\left.\mathrm{rr} C(H)^{0}\right)$ defined by $c \mapsto n c n^{-1}, c \in C(H)$, $n \in N(H)$. We say that a map $\phi: \tilde{M}^{H} \rightarrow C(H)$ (or $C(H)^{0}$ ) is $N(H)$-skew equivariant if $\phi$ is $N(H)$-equivariant relative to the $N(H)$-actions on $\tilde{M}^{H}$ and $C(H)$ (or $C(H)^{0}$ ). Suppose $f \in F^{r}(M)$. We say that $\gamma_{f}: \tilde{M}^{H} \rightarrow N(H) / H$ lifts to $C(H)$ if there exists a $C^{r} \operatorname{map}$ $\hat{\gamma}_{f}: \ddot{M}^{H}+C(H)$ such that $\alpha \hat{\gamma}_{f}=\gamma_{f}$. Even if $\gamma_{f}$ lifts to $\hat{\gamma}_{f}$ it need not generally be true that $\hat{\gamma}_{f}$ is $N(H)$-equivariant and so we cannot assert that the lifting of $\gamma_{f}$ is a sufficient condition for $f$ to lie in $\Gamma^{r}(M)$. It is, however, obviously a necessary condition. Suppose now that $f \in \hat{F}^{\prime}(M)$. Then $\Upsilon_{f}: \tilde{M}^{H} \rightarrow(N(H) / H)^{0}$. Since $(N(H) / H)^{0} \approx N(H)^{0} / H \cap N(H)^{0}$ we may regard $\gamma_{f}$ as a map into $N(H)^{0} / H \cap N(H)^{0}$. We say $\gamma_{f}$ lifts to $C(H)^{0}$ if there exists a $C^{r}$-map $\hat{\gamma}_{f}: \tilde{M}^{H}+C(H)^{0}$ such that $B \hat{\gamma}_{f}=\gamma_{f}$. Again the lifting of $\gamma_{f}$ to $C(H)^{0}$ is obviously a necessary condition for $f$ to lie in $\hat{\Gamma}^{r}(M)$.

PROPOSITION 3. Suppose $M$ is a compact G-manifold with all G-orbits of the same dimension and $H$ is a principal isotropy subgroup of the action of $G$ on $M$. Assume that $N(H)$ is connected and $H$ is finite. Let $f \in F^{r}(M)$. Then $\gamma_{f}$ lifts to $C(H)^{0}$ if and only if $f \in \hat{\Gamma}^{p}(M)$

Proof. Let $f \in F^{P}(M)$ and suppose $\gamma_{f}$ lifts to $C(H)^{0}$. Fix $x \in M^{\langle H\rangle}$. For all $n \in H(H)$ we have $f(n x)=n f(x)$ and so $\hat{\gamma}_{f}(n x) n x=\hat{\gamma}_{f}(x) x$. Therefore $\hat{\gamma}_{f}(x)^{-1} n^{-1} \hat{\gamma}_{f}(n x) n \in H$. The connectedness of $N(H)$ together with the finiteness of $B$ now implies that $\hat{\gamma}_{f}(x)^{-1} n^{-1} \hat{\gamma}_{f}(n x) n=e$. Hence $\hat{\gamma}_{f}$ is skew $N(B)$-equivariant. The converse is trivial. 
EXAMPLE. Let $M$ be a compact $S^{\perp}$-manifold with all isotropy groups finite. Denote the principal isotropy group of the action by $K$. Let $f \in F^{r}(M)$. Then $f \in \Gamma^{r}(M)$ if and only if $\gamma_{f}: M \rightarrow S^{l} / K$ lifts to $S^{l}$. In particular, $F^{r}(M) / \Gamma^{r}(M) \cong K$.

REMARK 1. Using the above ideas it is now easy to show that if $f \in F^{r}(M)$ is sufficiently $c^{0}$-close to the identity then $f \in \Gamma^{r}(M)$. Indeed, if $f$ is $c^{0}$ close to the identity so is $\gamma_{f}$ and so $\gamma_{f}$ lifts to $C(H)^{0}$. But now we can lift $\hat{\gamma}_{f}$ to $c(h)$ and average over $N(H)$ to obtain a skew $N(H)$-equivariant lifting of $\gamma_{f}$.

REMARK 2. Let $K^{r}(M)=\left\{x \in S^{r}(M): \hat{x}=\right.$ identity $\}$. Then $K^{r}(M)$ is a closed subgroup of $S^{r}(M), r \geq 0$. By Proposition 2, $F^{r}(M) \approx S^{r}\left(\tilde{M}^{H}\right)$. But $S^{r}\left(\tilde{H}^{H}\right)$ is the space of $C^{r} N(H)$-equivariant maps of $\tilde{M}^{H}$ to $N(H) / H$ where we take the $N(H)$-action on $N(H) / H$ defined by $k \mapsto n k n^{-1}$, $n \in N(H), k \in N(H) / H$. In particular, taking $r=\infty$, we see that $S^{r}\left(\tilde{M}^{H}\right)$ has the structure of a Fréchet Lie group (see Field [3] or Palais [7] for background on differential structures on spaces of smooth equivariant maps). Using the remarks preceding Proposition 3 together with the argument of the proof of Proposition 3 it is easily verified that $\Gamma^{\infty}(M)$ has the structure of a closed Fréchet Lie subgroup of $F^{\infty}(M)$. Since $S^{\infty}(M)$ may be represented as a space of smooth $G$-equivariant maps we see also that $S^{\infty}(M)$ has the structure of a Fréchet Lie group with closed Fréchet Lie subgroup $K^{\infty}(M)$. It now follows straightforwardly that the sequence

$$
1 \rightarrow K^{\infty}(M) \rightarrow S^{\infty}(M) \stackrel{p}{\rightarrow} \Gamma^{\infty}(M) \rightarrow 1,
$$

$p(x)=\hat{x}$, is a short exact sequence of Frechet Lie groups. Lemma $B$ implies that $p$ admits local smooth sections.

We now turn to the case where the dimension of G-orbits varies. Recall from Field [4] that associated to any compact $G$-manifold $M$ we have a compact $G \times\left(\mathbb{Z}_{2}\right)^{N-1}$-manifold $\hat{M}$ (the "resolution" of $M$ ) and 
projection $\pi: \hat{M} \rightarrow M$ satisfying:

(1) the action of $G$ on $\hat{M}$ is principal;

(2) $\pi$ is equivariant in the sense that

$$
\pi((g, \gamma) x)=g \pi(x), g \in G, \quad \gamma \in\left(\mathbb{Z}_{2}\right)^{N-1}
$$

(3) for $r \geq N-1$, there exists a continuous homomorphism

$$
\phi: \operatorname{Diff}_{G}^{p}(M) \rightarrow \operatorname{Diff} \underset{G \times\left(Z_{2}\right)^{N-1}}{(M)}
$$

such that $\pi \phi(f)=f \pi, f \in \operatorname{Diff}_{G}^{r}(M)$.

THEOREM 2. Let $f \in F_{0}^{\infty}(M)$. Then there exists a skew G-equivariant map $\mu \in S^{\infty}(\hat{M})$ such that

(1) $\phi(f)=\hat{\mu}$,

(2) $f(x)=\mu(y) x, \quad x \in M$ and $y$ any point in $\pi^{-1}(x)$.

Proof. If $f \in F_{0}^{\infty}(M)$ then $\phi(f) \in F_{0}^{\infty}(\hat{M})$. Since all $G \times\left(\mathbb{Z}_{2}\right)^{N-1}$ orbits have the same dimension, Theorem 1 implies that there exists $\mu \in S^{\infty}(M)$ such that $\phi(f)=\hat{\mu}$. Now $\pi \phi(f)=f^{\prime} \pi$ and so if $\pi(y)=x$ we have $f(x)=\mu(y) x$.

REMARK. Theorem 2 may be strengthened. If we let $q$ denote the number of orbit types of $M$ with Gorbits having the same dimension as the principal G-orbit type then we need only blow up $M(N-q)$-times to obtain a $G \times\left(\mathbb{Z}_{2}\right)^{N-q}$-manifold with all G-orbits of the same dimension. Using Property (3) of the resolution we see that the theorem is valid for $f \in E_{0}^{N-q+1}(M)$ rather than $E_{0}^{\infty}(M)$. Of course, we can apply Theorem 1 to each orbit type to find a skew G-equivariant map $\gamma: M \rightarrow G$ such that $f=\hat{x}$. But this approach tells us nothing about the singularities of $X$.

One consequence of Theorem 2 and Example 4 is that we need to modify the definition of G-structural stability for equivariant diffeomorphisms given in Field [5].

DEFINITION. Let $f \in \operatorname{Diff}_{G}^{p}(M)$. We say $f$ is $G$-structurally stable 
if we can find a neighbourhood $N$ of $f\left(c^{r}\right.$ topology) such that for all $j \in N$ there exists an equivariant homeomorphism $h$ of $M$ and skew $G$-equivariant map $Q: M \rightarrow G$ such that

$$
Q(f(x)) h(f(x))=j h(x) \text {, all } x \in M .
$$

REMARK. In Field [5] we required $Q$ to be continuous. By what we have shown above it is unrealistic to require $Q$ to be continuous unless, for example, all G-orbits have the same dimension. Note, however, that $Q \circ h: M \rightarrow M$ is always an equivariant homeomorphism even if $Q$ is not continuous.

We conclude with an analysis of $S^{1}$-manifolds with non-empty fixed point set. First an elementary technical lemma whose proof we include for completeness.

LEMMA 4. Let $f: \mathbb{C} \times \mathbf{R}^{k} \rightarrow \mathbb{C}$ be smooth and vanish at $(0,0)$. Suppose there exists a smooth function $\alpha(z, t)$ defined on $\left(\mathbf{C} \times \mathbb{R}^{k}\right) \backslash\{0,0\}$ such that $f(z, t) \doteq \alpha(z, t) z,(z, t) \neq 0$. Then extends smoothly to $\mathbb{C} \times \mathbf{R}^{k}$.

Proof. Fix $N \geq 1$. By Taylor's theorem

$$
f(z, t)=\left(\sum_{j=1}^{N}\left(\sum_{i_{1}=0}^{j} a_{i_{1} j-i_{1}}^{j}(t) z^{i_{1}}{ }^{j-i_{1}}\right)\right)+\sum_{i_{1}=0}^{N+1} a_{i_{1} N+1-i_{1}}^{N+1}(z, t) z^{i_{1}}{ }^{N+1-i_{1}}
$$

where the $a^{j}$ 's are smooth C-valued functions. Dividing by $z$ we see that

$$
\frac{f(z, t)}{z}=A(z, t)+\sum_{j=1}^{N} \frac{a^{j}(t) \bar{z}^{-j}}{z}+\frac{a^{N+1}(z, t) z^{-n+1}}{a},
$$

where $A, a^{1}, \ldots, a^{N+1}$ are smooth. We claim $a^{1}=\ldots=a^{N} \equiv 0$. If not, choose $p, 1 \leq p \leq N$, to be the smallest integer such that $a^{p}(t) \neq 0$. Then

$$
\frac{f(z, t)}{z}=A(z, t)+\frac{a^{p}(t) \vec{z}^{p}}{z}+\frac{\bar{z}^{p+1}}{z} Q(z, t),
$$

where $Q$ is smooth. Fixing $t \neq 0$, we see that 


$$
\frac{\partial^{p-1}}{\partial z^{p-1}}\left(\frac{f(z, t)}{z}\right)=(-1)^{p}(p-1) ! \frac{a^{p}(t) \bar{z}^{p}}{z^{p}}+\left(\frac{\bar{z}^{p+1}}{z^{p}} R(z, t)+T(z, t)\right) \text {, }
$$

where $R$ and $T$ are smooth functions on $\mathbf{C} \times \mathbf{R}^{k}$. Letting $\boldsymbol{z} \rightarrow 0$ we see that $a^{p}(t)\left(\bar{z}^{p} / z^{p}\right)$ does not converge unless $a^{p}(t)=0$. Since all the other terms do converge to definite limits as $z \rightarrow 0$ we see that $a^{p}(t)=0, t \neq 0$. Since $a^{p}(t)$ is continuous on $\mathbb{R}^{k}$ we have therefore shown that $a^{p} \equiv 0$. Therefore $a^{l}=\ldots=a^{N} \equiv 0$. Consequently, for any $N \geq 1$ we may write

$$
\frac{f(z, t)}{z}=A(z, t)+\frac{\bar{z}^{N+1}}{z} S(z, t),
$$

where $A$ and $S$ are smooth on $\mathbf{C} \times \mathbb{R}^{k}$. But the right hand side of this equation is $C^{N-1}$ on $\mathbf{C} \times \mathbf{R}^{k}$. Since $N$ was chosen arbitrarily it follows that $f(z, t) / z$ is smooth on $\mathbb{C} \times \mathbb{R}^{k}$.

THEOREM 3. Let $M$ be a compact $S^{1}$-manifold. Then $F_{0}^{\infty}(M)=\Gamma_{0}^{\infty}(M)$. Proof. Let $f \in F_{0}^{\infty}(M)$. By Theorems 1 and 2 there exists a skew $S^{l}$-equivariant map $\chi: M \rightarrow S^{l}$ such that $f=\hat{\mathrm{X}}$ and $\hat{\mathrm{X}}$ is smooth off the fixed point set of the $S^{l}$-action. We must show that we can require $X$ to be smooth on all of $M$. Let $x$ be a fixed point of the $S^{l}$-action on $M$. Choosing slices at $x$ and $f(x)$ we easily reduce to showing that if $\mathbb{C}^{k}$ is an $S^{l}$-representation with no trivial factors and $f \in F^{\infty}\left(\mathbb{C}^{k}\right)$ then $f=\hat{x}$ for some $x \in S^{\infty}\left(c^{k}\right)$. By Theorems 1 and 2 , we may find a smooth skew $S^{\perp}$ equivariant map $\theta: \mathbb{C}^{k} \times\{0\} \rightarrow S^{l}$ such that

$$
f\left(z_{1}, \ldots, z_{k}\right)=\left(\theta(z)^{p_{1}} z_{1}, \ldots, \theta(z)^{p_{k_{2}}}\right), \quad z=\left(z_{1}, \ldots, z_{k}\right) \neq 0 .
$$

(Here the integers $p_{1}, \ldots, p_{k}$ are just the orders of the irreducible factors of the $S^{1}$-representation on $\left.\mathbb{C}^{k}.\right)$ By Lemma $4, \theta$ extends to a smooth map defined on all of $\mathbb{C}^{k}$. 


\section{References}

[1] Richard L. Bishop and Richard J. Crittenden, Geometry of manifolds (Pure and Applied Mathematics, 15. Academic Press, New York and London, 1964).

[2] Glen E. Bredon, Introduction to compact transformation groups (Pure and Applied Mathematics, 46. Academic Press, New York and London, 1972).

[3] Mike Field, "Equivariant dynamical systems", Bulz. Amer. Math. Soc. 76 (1970), 1314-1318.

[4] M.J. Field, "Resolving actions of compact Lie groups", BulZ. Austral. Math. Soc. 18 (1978), 243-254.

[5] M.J. Field, "Equivariant dynamical systems", Trans. Amer. Math. Soc. $259(1980), 185-205$.

[6] M.J. Field, "Isotopy and stability of equivariant diffeomorphisms", Proc. London Math. Soc. (to appear).

[7] Richard S. Palais, "The principle of symmetric criticality", Comm. Math. Phys. 69 (1979), 19-30.

[8] Gerald W. Schwarz, "Smooth functions invariant under the action of a compact Lie group", Topology 14 (1975), 63-68.

[9] Gerald W. Schwarz, "Lifting smooth homotopies of orbit spaces", Inst. Hautes Études Sci. Publ. Math. 51 (1980), 37-135.

[10] Arthur G. Wassermann, "Equivariant differential topology", Topology 8 (1969), 127-150.

Department of Pure Mathematics,

University of Sydney,

Sydney,

New South Wales 2006,

Australia. 